\title{
Euclidean Mahler measure and twisted links
}

\author{
DANIEL S SILVER \\ AlEXANDER STOIMENOW \\ SUSAN G WILLIAMS
}

\begin{abstract}
If the twist numbers of a collection of oriented alternating link diagrams are bounded, then the Alexander polynomials of the corresponding links have bounded euclidean Mahler measure (see Definition 1.2). The converse assertion does not hold. Similarly, if a collection of oriented link diagrams, not necessarily alternating, have bounded twist numbers, then both the Jones polynomials and a parametrization of the 2variable Homflypt polynomials of the corresponding links have bounded Mahler measure.
\end{abstract}

$57 \mathrm{M} 25 ; 37 \mathrm{~B} 40$

\section{Introduction}

If $f(t)$ is a nonzero polynomial with complex coefficients,

$$
f(t)=b \cdot \prod_{i=1}^{n}\left(t-\alpha_{i}\right) \in \mathbb{C}[t],
$$

then its Mahler measure [18] is

$$
M(f)=|b| \cdot \prod_{i=1}^{n} \max \left\{\left|\alpha_{i}\right|, 1\right\} .
$$

By convention, the Mahler measure of the zero polynomial is defined to be 0 . A well-known theorem of Kronecker implies that a monic (ie, $|b|=1$ ) integer polynomial has Mahler measure 1 if and only if it is a product of monomials and cyclotomic polynomials.

In 1933, D H Lehmer discovered a monic integer polynomial of degree 10,

$$
L(t)=t^{10}+t^{9}-t^{7}-t^{6}-t^{5}-t^{4}-t^{3}+t+1,
$$

with a single zero of modulus greater than 1, equal approximately to 1.17628 [16]. He asked whether given any $\epsilon>0$ there exists an integer polynomial $f(t)$ such that 
$1<M(f)<1+\epsilon$. Lehmer's question remains open. In fact, no integer polynomial has been found with Mahler measure less than $M(L)$ but greater than 1 .

Jensen's formula motivates a definition of Mahler measure for polynomials of more than one variable [18]. For any nonzero polynomial $f\left(t_{1}, \ldots, t_{d}\right)$ with complex coefficients, the Mahler measure of $f$ is defined by the following integral, which is possibly singular but nevertheless convergent:

$$
M(f)=\exp \int_{0}^{1} \cdots \int_{0}^{1} \log \left|f\left(e^{2 \pi i \theta_{1}}, \ldots, e^{2 \pi i \theta_{d}}\right)\right| d \theta_{1} \cdots d \theta_{d} .
$$

Lehmer's question for polynomials of any higher degree is equivalent to the question for 1-variable polynomials (Boyd [2]). The reader who is interested in additional background information on Mahler measure is encouraged to consult Everest and Ward [9].

Remark 1.1 The Mahler measure of a nonzero Laurent polynomial $t^{-r} f(t), f(t) \in$ $\mathbb{C}[t]$, is defined to be $M(f)$. Likewise, we define the Mahler measure of $t^{1 / 2} f(t)$ to be $M(f)$. Thus the Mahler measure of both the 1-variable Alexander and the Jones polynomial of any oriented knot or link is well defined. Similarly one deals with several variable Laurent polynomials.

A topological interpretation of Mahler measures of single- and multivariable-Alexander polynomials of links in terms of homology growth of branched covers was given by Silver and Williams [26]. Moreover, the Mahler measure of the Alexander polynomial of a fibered link is a suitably defined growth rate of Lefschetz numbers of the fiber monodromy [25]. Slowing down the dynamics of pseudo-Anosov homeomorphisms is equivalent to Lehmer's question. In this sense, it is a question about fibered knots and links and the dynamical properties of their monodromies.

Lehmer's polynomial $L(t)$ occurs (up to the interchange $t \leftrightarrow-t$ ) as the Alexander polynomial of a knot. Perhaps the most interesting such knot is the $(-2,3,7)$-pretzel knot, a fibered hyperbolic knot with noteworthy properties (see for example Gordon [10], Hironaka [11]). If Lehmer's question has an affirmative answer, then the polynomial $f(t)$ can be chosen to be a (1-variable) Alexander polynomial of a fibered hyperbolic arborescent (or Conway-algebraic) 2-component link in $S^{3}$ (Stoimenow [28]) or a fibered hyperbolic knot in a lens space $L(n, 1)$ [25]. (For a knot in $S^{3}$ one needs one of the conditions $f(1)= \pm 1$, whose meaning in Lehmer's question is unclear so far.)

Callahan, Dean and Weeks argue in [4] that for hyperbolic knots, the adjective "simple" should reflect geometric properties. Consequently, they propose that a hyperbolic knot or link should be considered simple if its complement can be constructed with relatively few 
ideal tetrahedra. Examples in [27] suggest that the multivariable Alexander polynomial of such a knot or link has small Mahler measure. Examples of simple hyperbolic knots in [6] suggest a similar statement for the Jones polynomial $V_{\ell}$. The $(-2,3,7)$-pretzel knot is one example, with complement composed of only 3 ideal tetrahedra.

Often the logarithm of Mahler measure arises as the topological entropy of algebraic dynamical systems. In [17] Lind and Ward defined $p$-adic and euclidean entropy for automorphisms of generalized solenoids, the type of algebraic dynamical systems that arise in [26], and they showed that topological entropy is their sum. The two components correspond to the two contributions in the definition of Mahler measure of a polynomial $f(t)$, one from the leading coefficient of $f$ and the other from its zeros. This motivates the following.

Definition 1.2 If $f(t) \in \mathbb{C}[t]$ has zeros $\alpha_{1}, \ldots, \alpha_{n}$, then its euclidean Mahler measure is

$$
M_{e}(f)=\prod_{i=1}^{n} \max \left\{\left|\alpha_{i}\right|, 1\right\} .
$$

Remark 1.3 Euclidean Mahler measure of the Alexander polynomial of a knot $k$ is a natural quantity. The homology group $H_{1}(\tilde{X} ; \mathbb{R})$ of the infinite cyclic cover of $k$ with real coefficients is a finite-dimensional vector space, and a generator of the deck transformation group of $\tilde{X}$ induces an automorphism. It is not difficult to see that the product of moduli of those eigenvalues that are outside the unit circle coincides with $M_{e}\left(\Delta_{k}\right)$.

Our main result is concerned with sequences of knots or links that have 1-variable Alexander polynomials with euclidean Mahler measures tending to infinity or Jones polynomials with Mahler measures tending toward infinity. In such cases we can conclude that the twist numbers also tend toward infinity. In the case that the knots or links are alternating and hyperbolic, their volumes increase without bound. (Dasbach and Lin [8] give an expression of the twist number of alternating diagrams in terms of the Jones polynomial, which gives a different relation between twist number and volume.)

Acknowledgements The authors are grateful to Hiroshima University and Osaka City University for their hospitality while this work was performed. Their visits were funded by the program "Constitution of wide-angle mathematical basis focused on knots," directed by Professor Akio Kawauchi and part of the 21st Century COE Program. The calculations were aided by Knot, a program developed by K Kodama [14]. The first and third authors were partially supported by NSF grant DMS-0304971, and the second author was supported by JSPS Postdoc grant P04300. 


\section{Statement of main theorem}

By full-twisting an oriented link $\ell$, we mean cutting a pair of adjacent arcs of a diagram $D$ for $\ell$, inserting some number $q$ of full twists (right-handed if $q$ is positive and left-handed otherwise) and then reattaching the arcs. In this way, we obtain a sequence of links $\ell_{q}$. As $q$ goes to infinity, the Mahler measures of the Jones polynomials $V_{\ell_{q}}$ converge (Champanerkar and Kofman [5]); the Mahler measures of the Alexander polynomials $\Delta_{\ell_{q}}$ will also converge, provided that the twisted arcs are coherently oriented [27]. If we insert arbitrary numbers $q_{i}$ of full-twists at several sites, then as all $q_{i}$ grow without bound, we again we have convergence. However, the set of Mahler measures produced will generally have infinitely many distinct limit points (cf Example 5.1). Theorem 2.1 implies that the limit points are bounded.

In the case that the arcs being twisted are not coherently oriented, the Mahler measures of the Alexander polynomials of $\ell_{q}$ can grow without bound. Twist knots provide simple examples. However, we will see that if the diagram $D$ is alternating and we replace Mahler measure by euclidean Mahler measure, then again limits exist and limit points are bounded.

In order to state the main result, we need the following notions.

Let $D$ be a link diagram. A bigon region is a complementary region with exactly two crossings in its boundary. A twist is either a connected row of bigon regions, maximal in the sense that it is not part of a longer row of bigons, or else it is a single crossing that is adjacent to no bigon region. The number of twists of $D$ is called the twist number, denoted by $t(D)$. The twist number of a link $\ell$ is the minimal twist number of all of its diagrams.

A trivial split component of a link diagram is a circle (with no self-crossings) disjoint from the rest of the diagram. A trivial split component of a link is an unknotted component separable by a hyperplane from the rest of the link.

We define the length $\|p\|$ of a multivariable polynomial $p$ to be the sum of the absolute values of its coefficients. It follows from the triangle inequality and monotonicity of the $\log$ function that $M(p) \leq\|p\|$. (The inequality for single-variable polynomials appears in Everest and Ward [9] as an exercise. For another argument, see [31, Lemma $6.1]$ for $p \in \mathbb{Z}[x]$. The same proof works for $p \in \mathbb{R}[x]$, and as remarked there also for polynomials in several variables.)

Let $P_{\ell}(v, z)$ be the Homflypt skein polynomial with the skein relation

$$
v^{-1} P_{\ell_{+}}(v, z)-v P_{\ell_{-}}(v, z)=z P_{\ell_{0}}(v, z),
$$

Algebraic 83 Geometric Topology, Volume 6 (2006) 
normalized in the usual way so that the polynomial of the trivial knot is 1 . We will consider a 2-variable parametrization

$$
\widehat{P}_{\ell}(v, t)=P_{\ell}\left(v, t^{1 / 2}-t^{-1 / 2}\right)
$$

that is related by a variable change preserving Mahler measure and length to the polynomial $X(q, \lambda)$ that appears in Jones [12]. The Jones polynomial is obtained by setting $q=\lambda=t$, or in other words:

$$
V_{\ell}(t)=\widehat{P}_{\ell}(t, t)=P_{\ell}\left(t, t^{1 / 2}-t^{-1 / 2}\right),
$$

and the Alexander polynomial (with a particular choice of normalization) by

$$
\Delta_{\ell}(t)=\widehat{P}_{\ell}(1, t)=P_{\ell}\left(1, t^{1 / 2}-t^{-1 / 2}\right) .
$$

Here and below the term "Alexander polynomial" will refer to the one-variable version; its several-variable relative will be referred to as the "multi-variable Alexander polynomial." In each case, the polynomial is defined up to multiplication by a unit.

Theorem 2.1 (1) If $\mathcal{D}$ is a set of oriented link diagrams with twist numbers at most $n$, and no trivial split components, then the sets of lengths

$$
\left\{\left\|(t+1)^{n} V_{\ell(D)}(t)\right\| \mid D \in \mathcal{D}\right\}
$$

and

$$
\left\{\left\|\left(v^{2}-1\right)^{n}(t+1)^{n} \hat{P}_{\ell(D)}(v, t)\right\| \mid D \in \mathcal{D}\right\}
$$

are bounded.

(2) If $\mathcal{D}$ is a set of oriented alternating link diagrams with bounded twist numbers, then the set of euclidean Mahler measures $\left\{M_{e}\left(\Delta_{\ell(D)}\right) \mid D \in \mathcal{D}\right\}$ is bounded.

Corollary 2.2 If $\mathcal{D}$ is a set of oriented link diagrams with bounded twist number, the set of Mahler measures $\left\{M\left(V_{\ell(D)}\right) \mid D \in \mathcal{D}\right\}$ and $\left\{M\left(\widehat{P}_{\ell(D)}\right) \mid D \in \mathcal{D}\right\}$ of the Jones and parametrized Homflypt polynomials are bounded.

Proof Corollary 2.2 follows from the fact that the Mahler measure of a polynomial is bounded by its length, while multiplying a polynomial by a cyclotomic polynomial does not change its Mahler measure.

Corollary 2.3 If $\ell_{q}$ is a sequence of prime alternating hyperbolic links such that either $M\left(V_{\ell_{q}}\right)$ or $M_{e}\left(\Delta_{\ell_{q}}\right)$ increases without bound, then $\operatorname{Volume}\left(S^{3} \backslash \ell_{q}\right)$ also increases without bound. 
Proof Each link $\ell_{q}$ admits a connected alternating diagram $D_{q}$ that is prime in the sense that any simple closed curve in the plane meeting $D_{q}$ in exactly two points disjoint from the crossings bounds a region containing no crossings. Theorem 1 of Lackenby [15] implies that

$$
v_{3}\left(t\left(D_{q}\right)-2\right) / 2 \leq \operatorname{Volume}\left(S^{3} \backslash \ell_{q}\right),
$$

where $v_{3}(\approx 1.01494)$ is the volume of a regular hyperbolic ideal 3-simplex. Since the twist numbers $t\left(D_{q}\right)$ increase without bound, by Theorem 2.1, the volumes of $S^{3} \backslash \ell_{q}$ also increase without bound.

\section{Twisting}

We prepare for the proof of Theorem 2.1 by establishing results about the Alexander, Jones and skein polynomials of an oriented link when twists at several sites are performed.

By a wiring diagram we mean a planar diagram consisting of $n$ vertices or twist sites $v_{1}, \ldots, v_{n}$ of the three possible types shown in Figure 1, connected by oriented arcs. A vertex of the first type is a parallel twist site while a vertex of the second or third type is anti-parallel. We call the number of twist sites the order of the wiring diagram.

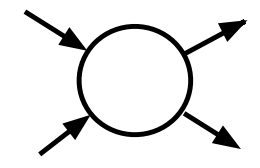

(1)

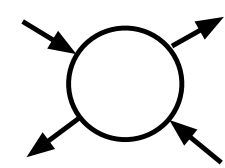

(2)

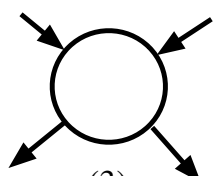

(3)

Figure 1: Vertex types of a wiring diagram

Given a wiring diagram of order $n$, we consider $n$-tuples $\left(q_{1}, \ldots, q_{n}\right)$, where $q_{i} \neq \infty$ if $v_{i}$ is of the first type; $q_{i}$ is even if $v_{i}$ is of the second type; and $q_{i}$ is odd if $v_{i}$ is of the third type. We obtain an oriented link $\ell\left(q_{1}, \ldots, q_{n}\right)$ by replacing the vertex $v_{i}$ by $T_{-q_{i}}$ if $v_{i}$ is of the first type, and $T_{q_{i}}$ if it is of the second or third type. (This choice assures that the sign of $q_{i}$ agrees with the sign of the crossings at that site in the resulting link.) We will use the previous term twist to refer to each embedded tangle $T_{q_{i}}$. Clearly any oriented link diagram with twist number $n$ can be described by a wiring diagram of order $n$. Note that a twist consisting of a single crossing can be regarded as either parallel or anti-parallel, depending on the choice of wiring diagram. 


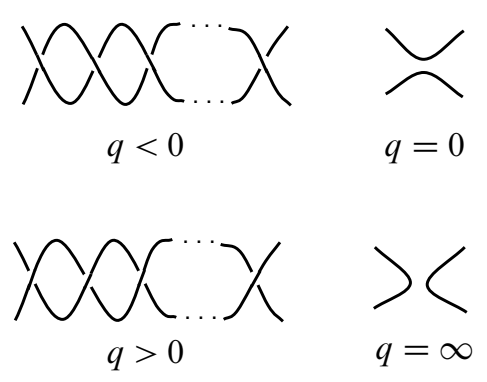

Figure 2: Tangles $T_{q}$

Lemma 3.1 Let $D$ be an oriented link diagram, and let $D_{q}$ (for $q \in \mathbb{Z}$ ) be the diagram that results from replacing a pair of adjacent parallel arcs of $D$ by the tangle $T_{-q}$ in such a way that $D_{0}=D$. Let $\ell_{q}$ be the links described by $D_{q}$. Then

$$
\begin{gathered}
(t+1) \Delta_{\ell_{q}}(t)=\left(\Delta_{\ell_{0}}(t)+t^{1 / 2} \Delta_{\ell_{1}}(t)\right) t^{q / 2}+(-1)^{q}\left(t \Delta_{\ell_{0}}(t)-t^{1 / 2} \Delta_{\ell_{1}}(t)\right) t^{-q / 2}, \\
(t+1) V_{\ell_{q}}(t)=\left(V_{\ell_{0}}(t)+t^{-1 / 2} V_{\ell_{1}}(t)\right) t^{3 q / 2}+(-1)^{q}\left(t V_{\ell_{0}}(t)-t^{-1 / 2} V_{\ell_{1}}(t)\right) t^{q / 2}, \\
(t+1) \widehat{P}_{\ell_{q}}(v, t)=v^{q}\left[\left(\widehat{P}_{\ell_{0}}(v, t)+v^{-1} t^{1 / 2} \widehat{P}_{\ell_{1}}(v, t)\right) t^{q / 2}+\right. \\
\left.(-1)^{q}\left(t \widehat{P}_{\ell_{0}}(v, t)-v^{-1} t^{1 / 2} \widehat{P}_{\ell_{1}}(v, t)\right) t^{-q / 2}\right] .
\end{gathered}
$$

Proof Let $D_{q}$ be the diagram with $q$ twists. Set

$$
p_{q}(v, t)=v^{-\omega\left(D_{q}\right)} \hat{P}_{D_{q}}(v, t),
$$

where $\omega\left(D_{q}\right)$ is the writhe. From the skein relation

$$
v^{-1} P_{+}(v, z)-v P_{-}(v, z)=z P_{0}(v, z),
$$

we have

$$
p_{q+2}(v, t)-p_{q}(v, t)=\left(t^{1 / 2}-t^{-1 / 2}\right) p_{q+1}(v, t) .
$$

For the case $q \geq 0$ we use the generating function

$$
f(v, t, x)=\sum_{q=0}^{\infty} p_{q}(v, t) x^{q} .
$$

Multiplying equation (3-1) by $x^{q}$ and summing from 0 to $\infty$ gives

$$
\frac{f(v, t, x)-p_{0}(v, t)-p_{1}(v, t) x}{x^{2}}-f(v, t, x)=\left(t^{1 / 2}-t^{-1 / 2}\right) \frac{f(v, t, x)-p_{0}(v, t)}{x},
$$


which can be written as

$$
f(v, t, x)=\frac{p_{0}(v, t)+\left(p_{1}(v, t)-\left(t^{1 / 2}-t^{-1 / 2}\right) p_{0}(v, t)\right) x}{1-\left(t^{1 / 2}-t^{-1 / 2}\right) x-x^{2}} .
$$

A partial fraction decomposition gives

$$
f(v, t, x)=\frac{1}{t+1}\left[\frac{p_{0}(v, t)+t^{1 / 2} p_{1}(v, t)}{1-t^{1 / 2} x}+\frac{t p_{0}(v, t)-t^{1 / 2} p_{1}(v, t)}{1+t^{-1 / 2} x}\right] .
$$

Taking series expansions in $x$ of these rational functions, and equating coefficients, shows that

$p_{q}(v, t)=\frac{1}{t+1}\left[\left(p_{0}(v, t)+t^{1 / 2} p_{1}(v, t)\right) t^{q / 2}+(-1)^{q}\left(t p_{0}(v, t)-t^{1 / 2} p_{1}(v, t)\right) t^{-q / 2}\right]$.

Since $\omega\left(D_{q}\right)=q+\omega\left(D_{0}\right)$, we see the statement for $\widehat{P}$. The remaining results follow by setting $v=1$ for the Alexander polynomial, and setting $v=t$ for the Jones polynomial. The case $q<0$ is handled in the same manner, using the generating function $g(v, t, x)=\sum_{r=0}^{\infty} p_{1-r}(v, t) x^{r}$ and then setting $r=1-q$.

Remark 3.2 (1) Champanerkar and Kofman showed in [5] using Jones-Wenzl idempotents that for full twists, $V_{\ell_{q}}(t)$ can be expressed as a rational function of $t$ and $t^{q}$. In fact, their result holds more generally for $q$ full twists on any number of strands (with arbitrary orientation).

(2) A special case of the first formula of Lemma 3.1 appears in Bhatty [1].

Lemma 3.3 Let $D$ be an oriented link diagram, and let $D_{q}$ (for $q \in 2 \mathbb{Z} \cup\{\infty\}$ ) be the diagram that results from replacing a pair of adjacent anti-parallel arcs of $D$ by the tangle $T_{q}$ in such a way that $D_{0}=D$. Let $\ell_{q}$ be the link described by $D_{q}$. Then for $q \in 2 \mathbb{Z}$,

$$
\begin{gathered}
\Delta_{\ell_{q}}(t)=\Delta_{\ell_{0}}(t)+\frac{q}{2}\left(t^{1 / 2}-t^{-1 / 2}\right) \Delta_{\ell_{\infty}}(t) \\
(t+1) V_{\ell_{q}}(t)=\left((t+1) V_{\ell_{0}}(t)+t^{1 / 2} V_{\ell_{\infty}}(t)\right) t^{q}-t^{1 / 2} V_{\ell_{\infty}}(t), \\
P_{\ell_{q}}(v, z)=\frac{v^{q}-1}{v-v^{-1}} z P_{\ell_{\infty}}(v, z)+v^{q} P_{\ell_{0}}(v, z)
\end{gathered}
$$

Lemma 3.3 is a straightforward consequence of the Homflypt skein relation. (See formula (8) in [30], or formula (4) in [29], including a correction of the misprint in the first reference.) Note that the case of an odd number of anti-parallel twists can be handled by letting $D=D_{0}$ have a single crossing at the twist site in question. 
Theorem 3.4 Consider a wiring diagram of order $n$, and let $\ell\left(q_{1}, \ldots, q_{n}\right)$ be the oriented link defined at the beginning of the section. Assume that sites $1, \ldots, m$ are anti-parallel, and the remaining sites are parallel.

(1) The Jones polynomial of $\ell\left(q_{1}, \ldots, q_{n}\right)$ satisfies:

$$
(t+1)^{n} V_{\ell\left(q_{1}, \ldots, q_{n}\right)}(t)=W\left(t^{1 / 2}, t^{q_{1} / 2}, \ldots, t^{q_{n} / 2}\right),
$$

where $W\left(u, w_{1}, \ldots, w_{n}\right) \in \mathbb{Z}\left[u^{ \pm 1}, w_{1}^{ \pm 1}, \ldots, w_{n}^{ \pm 1}\right]$ depends only on the wiring diagram describing $\ell\left(q_{1}, \ldots, q_{n}\right)$ and the parities of $q_{1}, \ldots, q_{n}$.

(2) The Alexander polynomial of $\ell\left(q_{1}, \ldots, q_{n}\right)$ satisfies:

$$
(t+1)^{n-m} \Delta_{\ell\left(q_{1}, \ldots, q_{n}\right)}(t)=\sum \frac{q_{1}^{\delta_{1}} \cdots q_{m}^{\delta_{m}}}{2^{m}} X_{\delta_{1}, \ldots, \delta_{n}}\left(t^{1 / 2}, t^{q_{m+1} / 2}, \ldots, t^{q_{n} / 2}\right),
$$

where the sum ranges over all choices of $\delta_{i} \in\{0,1\}, i=1, \ldots, n$, and each $X_{\delta_{1}, \ldots, \delta_{n}} \in$ $\mathbb{Z}\left[u^{ \pm 1}, w_{1}^{ \pm 1}, \ldots, w_{n-m}^{ \pm 1}\right]$ depends only on the wiring diagram describing $\ell\left(q_{1}, \ldots, q_{n}\right)$ and the parities of the $q_{1}, \ldots q_{n}$.

(3) The Homflypt polynomial of $\ell\left(q_{1}, \ldots, q_{n}\right)$ satisfies:

$$
\begin{aligned}
& (t+1)^{n-m}\left(v^{2}-1\right)^{m} \hat{P}_{\ell\left(q_{1}, \ldots, q_{n}\right)}(v, t) \\
& \quad=Y\left(v^{1 / 2}, t^{1 / 2}, v^{q_{1} / 2}, \ldots, v^{q_{m} / 2}, t^{q_{m+1} / 2}, \ldots, t^{q_{n} / 2}\right),
\end{aligned}
$$

where $Y\left(u, w, u_{1}, \ldots, u_{m}, w_{m+1}, \ldots, w_{n}\right) \in \mathbb{Z}\left[u^{ \pm 1}, w^{ \pm 1}, \ldots, w_{n}^{ \pm 1}\right]$ depends only on the wiring diagram describing $\ell\left(q_{1}, \ldots, q_{n}\right)$ and the parities of $q_{1}, \ldots, q_{n}$.

Proof Using either Lemma 3.1 (if $v_{n}$ is parallel) or Lemma 3.3 (if $v_{n}$ is antiparallel), we can write $V_{\ell\left(q_{1}, \ldots, q_{n}\right)}$ as a polynomial in $t^{1 / 2}, t^{q_{n} / 2}$ and the expressions $V_{\ell\left(q_{1}, \ldots, q_{n-1}, 0\right)}(t), V_{\ell\left(q_{1}, \ldots, q_{n-1}, 1\right)}(t)$ and $V_{\ell\left(q_{1}, \ldots, q_{n-1}, \infty\right)}(t)$ (only two of the expressions will appear). The polynomial depends only on the parity of $q_{n}$. Continuing in this fashion with each twist site in turn, we write $V_{\ell\left(q_{1}, \ldots, q_{n}\right)}$ as a polynomial in $t^{1 / 2}, t^{q_{1} / 2}, \ldots, t^{q_{n} / 2}$ and the polynomials $V_{\ell\left(\epsilon_{1}, \ldots, \epsilon_{n}\right)}(t) \in \mathbb{Z}\left[t^{ \pm 1 / 2}\right]$, where $\epsilon_{i} \in\{0,1, \infty\}$. This polynomial has the desired form, and depends only on the wiring diagram and the parities of $q_{1}, \ldots, q_{n}$. The argument for the Homflypt polynomial is similar.

We prove the second assertion of Theorem 3.4. For $i=1, \ldots, m$, we set $\delta_{i}=1$ if $\epsilon_{i}=\infty$ and $\delta_{i}=0$ if $\epsilon_{i}=0$ ( $q_{i}$ even) or $\epsilon_{i}=1$ ( $q_{i}$ odd). Using Lemma 3.3 we can write

$$
\Delta_{\ell\left(q_{1}, \ldots, q_{n}\right)}(t)=\sum\left\lfloor\frac{q_{1}}{2}\right\rfloor^{\delta_{1}} \ldots\left\lfloor\frac{q_{m}}{2}\right\rfloor^{\delta_{m}} \Delta_{\ell\left(\epsilon_{1}, \ldots, \epsilon_{m}, q_{m+1}, \ldots, q_{n}\right)}(t) .
$$


Now we use Lemma 3.1 as before to write $\Delta_{\ell\left(\epsilon_{1}, \ldots, \epsilon_{m}, q_{m+1}, \ldots, q_{n}\right)}(t)$ as a linear combination of polynomials in $t^{1 / 2}, t^{q_{m+1} / 2}, \ldots, t^{q_{n} / 2}$, that depend on the parity of $q_{m+1}, \ldots, q_{n}$.

Theorem 3.4 has independent interest. It says that if a collection of links has bounded twist number (and no trivial split components), then the result of multiplying either the Jones or Alexander polynomials by a fixed polynomial (a power of $t+1$ ) is a collection of polynomials with a bounded number of nonzero coefficients. Lemma 3.5 shows that a collection of polynomials $f g^{N}$ will have such a form only in trivial cases.

Lemma 3.5 If $f(t), g(t) \in \mathbb{Z}\left[t^{ \pm 1}\right], f(t) \neq 0, g(t)$ not a unit, then the number of nonzero coefficients of $\mathrm{fg}^{N}$ tends to infinity as $N$ increases without bound.

Proof We may assume without loss of generality that $f(t)=1+a_{1} t+\cdots$ and $g(t)=1+b_{m} t^{m}+\cdots$, where $a_{i}, b_{i} \in \mathbb{Z}$ and $b_{m} \neq 0$. We show that for each $k \neq 0$, the coefficient $c_{k m}^{(N)}$ of $t^{k m}$ in $f g^{N}$ is nonzero for all sufficiently large $N$. This coefficient is given by the sum

$$
c_{k m}^{(N)}=\sum_{n+n_{1}+\cdots+n_{N}=k m} a_{n} b_{n_{1}} \cdots b_{n_{N}},
$$

with $n, n_{i} \geq 0$. Splitting the sum over the number $p$ of nonzero $n_{i}$, we can write it as

$$
c_{k m}^{(N)}=\sum_{p=1}^{k}\left(\begin{array}{c}
N \\
p
\end{array}\right) \sum a_{n} b_{n_{1}} \cdots b_{n_{p}},
$$

where the second summation is taken over all $n, n_{1}, \ldots, n_{p}$ with $m \leq n_{i}, i=1, \ldots, p$, and $n+n_{1}+\cdots+n_{p}=k m$. The second summation is independent of $N$ for each $p$, so for $N \geq k$ we may regard $c_{k m}^{(N)}$ as a polynomial in $N$. The leading term of the polynomial comes from the summand corresponding to $p=k$, and it is given by $\frac{1}{k !} b_{m}^{k} N^{k}$. Hence $c_{k m}^{(N)}$ is nonzero for $N$ sufficiently large.

Corollary 3.6 If $\ell$ is a link with non-trivial Jones or Alexander polynomial (ie, not equal to 1$)$ and no trivial split components, then the twist number of the connected sum $\ell \sharp \ell \sharp \cdots \sharp \ell$ tends to infinity as the number of summands increases without bound.

Proof Let $g$ denote either the Jones or Alexander polynomial of $\ell$. Since $g$ is non-trivial, it is not a unit. For the Alexander polynomial, this is a consequence of normalization, as a specialization of $P$ (see paragraph preceding Theorem 2.1). In the case of the Jones polynomial, it follows from the properties given in section 12 of [12]. 
The Jones and Alexander polynomials of $\sharp_{i=1}^{N} \ell$ have the form $g^{N}$. If the connected sums have bounded twist numbers, then after multiplying by a suitable power $f(t)$ of $t+1$, the polynomials $\mathrm{fg}^{N}$ have a bounded number of nonzero coefficients, contradicting Lemma 3.5.

The following proposition will be needed for the proof of Theorem 2.1.

Proposition 3.7 Let $f\left(x, y_{1}, \ldots, y_{n}\right) \in \mathbb{Z}\left[x^{ \pm 1}, y_{1}^{ \pm 1}, \ldots, y_{n}^{ \pm 1}\right]$. There is a constant $C$ with

$$
\left\|f\left(x, x^{q_{1}}, \ldots, x^{q_{n}}\right)\right\| \leq C,
$$

for all $\left(q_{1}, \ldots, q_{n}\right) \in \mathbb{Z}^{n}$.

Proof Write $f\left(x, y_{1}, \ldots, y_{n}\right)$ as the sum of terms $f_{j}(x) g_{j}\left(y_{1}, \ldots, y_{n}\right), 1 \leq j \leq m$, where $g_{j}$ is a product of powers of the $y_{i}$. Then

$$
\left\|f_{j}(x) g_{j}\left(x^{q_{1}}, \ldots, x^{q_{n}}\right)\right\|=\left\|f_{j}(x)\right\| .
$$

Hence we have

$$
\left\|f\left(x, x^{q_{1}}, \ldots, x^{q_{n}}\right)\right\| \leq \sum_{j=1}^{m}\left\|f_{j}(x)\right\|,
$$

from the triangle inequality.

The proof of Theorem 2.1 also requires some facts about Murasugi products. Consider an oriented diagram $D$ for a link $\ell$. By smoothing crossings according to the wellknown algorithm of Seifert [23], we obtain a number of (possibly nested) Seifert disks in the plane. An orientable spanning surface for the link, called a canonical Seifert surface, is then seen by connecting the disks with half-twisted bands corresponding to the crossings of the diagram.

The boundary of a Seifert disk is called a Seifert circle. If $C$ is a Seifert circle, then it decomposes the plane into two closed regions $U, V$ meeting along $C$. We say that $C$ is separating if both $(U \backslash C) \cap D$ and $(V \backslash C) \cap D$ are non-empty, otherwise $C$ is non-separating. If $C$ is separating, then let $D_{1}$ and $D_{2}$ be the diagrams constructed from $D \cap U$ and $D \cap V$, filling gaps with arcs from $C$ where they are needed. We say that $D$ is a ${ }^{*}$-product (or Murasugi product) of $D_{1}$ and $D_{2}$, and write $D=D_{1} * D_{2}$.

A diagram $D$ is special if it does not decompose as a Murasugi product, in other words, if and only if it has no separating Seifert circle. A general oriented diagram $D$ can be decomposed along its separating Seifert circles into a product $D_{1} * \cdots * D_{r}$ of special diagrams. We call $D_{i}$ the special (Murasugi) factors of $D$. If $D$ is alternating, so are 
the $D_{i}$; they are special alternating diagrams. Notice that each twist of $D$ is contained in some factor. The diagram $D$ is connected if and only if each $D_{i}$ is connected. For additional background, the reader is advised to consult Murasugi [19; 20] or Cromwell [7].

We shall assume in the following that $D$ is a connected oriented alternating diagram, and $D_{1} * \cdots * D_{r}$ its decomposition into special alternating diagrams $D_{i}$. Let $\ell, \ell_{1}, \ldots, \ell_{r}$ be the links represented by $D, D_{1}, \ldots, D_{r}$, respectively. We will make use of the fact, noted in [19], that the leading coefficient of $\Delta_{\ell}(t)$, up to sign, is the product of the leading coefficients of $\Delta_{\ell_{1}}(t), \ldots, \Delta_{\ell_{r}}(t)$. The fact holds more generally when $D$ is a homogeneous diagram (Murasugi and Przytycki [21]).

Associated to an oriented alternating diagram $D$ for a link $\ell$ there is a graph $\Gamma$ obtained in the following manner. Checker-board color the regions of the diagram with black and white, and let the vertices of $\Gamma$ correspond to the black regions. Two vertices are connected by an edge for each crossing shared by the corresponding regions. Note that $\Gamma$ is planar, (generally) with multiple edges, and interchanging colors transforms $\Gamma$ into the dual graph.

When $D$ is a connected special alternating diagram, it is possible to checker-board color so that each white region has a Seifert circle as its boundary. Then the degree of each vertex of $\Gamma$ is even, and we can orient the edges in such a way that they alternate in and out as we travel around each vertex. Such an orientation is unique up to global orientation reversal. (Note that the graph is connected since by assumption $D$ is connected.) Fix a vertex $v$, called the root. The edges of any spanning tree $T \subset \Gamma$ can be uniquely oriented "toward the root" so that each vertex other than $v$ has exactly one outgoing edge.

Define $\iota(T)$ to be the number of edges of $T$ that disagree with the orientation of $\Gamma$. We call such edges incoherent, and the others coherent. We call $T$ incoherent (resp. coherent) if all its edges are incoherent (resp. coherent). In Murasugi and Stoimenow [22] it is shown that, up to units in $\mathbb{Z}\left[t^{ \pm 1 / 2}\right]$,

$$
\Delta_{\ell}(-t)=\sum_{T \subset \Gamma} t^{\iota(T)}
$$

where the summation is taken over all spanning trees of $\Gamma$. A particular consequence is that the computation is independent of the choice of root $v$. Note also that, since the degree of $\Delta_{\ell}(t)$ coincides with the Euler characteristic of the canonical Seifert surface, the leading (resp. trailing) coefficient of $\Delta_{\ell}(t)$ is (up to sign) the number of incoherent (resp. coherent) spanning trees in $\Gamma$, and in particular this number is non-zero. Since $\Delta_{\ell}(t)$ is reciprocal, both quantities coincide. We will for convenience work with coherent spanning trees.

Algebraic ${ }^{6} \mathcal{G}$ Geometric Topology, Volume 6 (2006) 


\section{Proof of Theorem 2.1}

It suffices to consider the collection $\mathcal{D}$ of all oriented link diagrams $D$ with twist number $t(D)$ no greater than an arbitrary integer $n$. Since any $D \in \mathcal{D}$ can be obtained from a wiring diagram $X$ of order $n$, Theorem 3.4(1) and (3) imply that the lengths of $(t+1)^{n} V_{\ell}(t)$ and $\left(v^{2}-1\right)^{m}(t+1)^{n-m} \widehat{P}_{\ell}(v, t)$ are bounded, where $m$ is the number of anti-parallel twist sites in $X$, and $\ell$ ranges over the links with diagrams associated to $X$. In the latter case, the set of lengths remains bounded when we multiply by $\left(v^{2}-1\right)^{m-n}(t+1)^{m}$. There are finitely many wiring diagrams of order $n$ with no trivial split components, and the first part of Theorem 2.1 is proved.

If we perform repeated parallel twisting at one or more sites of an oriented link diagram, then the Alexander polynomials of the resulting links have bounded Mahler measures by Lemma 3.1 together with the previously mentioned fact that $M(p) \leq\|p\|$, for any nonzero polynomial.

However, if we perform repeated anti-parallel twisting at one or more sites, then, as we will see later in Example 5.2, the euclidean Mahler measures (and hence the Mahler measures) of the Alexander polynomials can grow without bound. Alternating links have better behavior under such twisting, which we will explain next.

Consider a wiring diagram of order $n$. Let $D$ be the diagram for the link $\ell\left(q_{1}, \ldots, q_{n}\right)$, the sites $1, \ldots, m$ being anti-parallel, the remaining sites parallel.

The form of $(t+1)^{n-m} \Delta_{\ell\left(q_{1}, \ldots, q_{n}\right)}(t)$ is given by Theorem 3.4(2). As in the case of the Jones polynomial, we can see that the polynomials

$$
X_{\delta_{1}, \ldots, \delta_{n}}\left(t^{1 / 2}, t^{q_{m+1} / 2}, \ldots, t^{q_{n} / 2}\right)
$$

have bounded lengths. To show that the family of polynomials $\Delta_{\ell\left(q_{1}, \ldots, q_{n}\right)}(t)$ has bounded euclidean Mahler measure, it suffices to show that no coefficient can grow more rapidly as a function of $q_{1}, \ldots, q_{m}$ than the leading coefficient.

Fix $\left(q_{m+1}, \ldots, q_{n}\right) \in \mathbb{Z}^{n-m}$ and $\left(\delta_{m+1}, \ldots, \delta_{n}\right) \in\{0,1\}^{n-m}$, and let $S$ be the set of $m$-tuples $\delta=\left(\delta_{1}, \ldots, \delta_{m}\right) \in\{0,1\}^{m}$ for which

$$
X_{\delta}(t)=X_{\delta_{1}, \ldots, \delta_{n}}\left(t^{1 / 2}, t^{q_{m+1} / 2}, \ldots, t^{q_{n} / 2}\right)
$$

is non-zero. We will show that if $\delta$ is maximal in $S$ (in the sense that $\delta_{i}^{\prime} \geq \delta_{i}$, for all $i \leq m$, implies that $\left.\delta^{\prime}=\delta\right)$, then $X_{\delta}(t)$ and $\Delta_{\ell\left(q_{1}, \ldots, q_{n}\right)}(t)$ have the same degree.

First consider the case that $D$ is a special alternating diagram. We make use of the notation established above. Since we are concerned with the growth of coefficients as functions of $q_{1}, \ldots, q_{m}$, we may assume that each $q_{i}$ is at least 2 . One easily checks 
that any anti-parallel twist in $D$ corresponds to a multiple edge in $\Gamma$, while a parallel twist corresponds to a chain (that is, an edge subdivided by any number of vertices).

It is convenient to form a quotient graph $\bar{\Gamma}$ by identifying each multiple edge to a single bi-oriented edge, called an anti-parallel edge. Edges that are not anti-parallel are said to be ordinary. We say a tree $\bar{T}$ in $\bar{\Gamma}$ to be coherent if it has a coherent lift in $\Gamma$.

Regard $q_{1}, \ldots, q_{m}$ as variables, and consider a monomial $q_{1}^{\delta_{1}} \cdots q_{m}^{\delta_{m}}$ for which $\delta=$ $\left(\delta_{1}, \ldots, \delta_{m}\right)$ is maximal. The variables $q_{i}$ that appear (that is, those with exponent $\delta_{i}=1$ ) correspond to a subgraph $\bar{\Sigma}$ of anti-parallel edges in $\bar{\Gamma}$ that contains no cycle, and hence is a forest. For otherwise, the graph $\Gamma$ would have a cycle of multiple edges, as in Figure 3 , and the link $\ell_{\left(\delta_{1}, \ldots, \delta_{m}\right)}$, which is obtained by smoothing crossings, would be split. In that event, $\Delta_{\ell\left(\epsilon_{1}, \ldots, \epsilon_{m}, q_{m+1}, \ldots, q_{n}\right)}$ and hence $X_{\delta_{1}, \ldots, \delta_{m}}$ would vanish, contradicting the assumption that the monomial $q_{1}^{\delta_{1}} \cdots q_{m}^{\delta_{m}}$ appears.

By equation (3-2) contributions to the leading coefficients of $\Delta_{\ell\left(q_{1}, \ldots, q_{n}\right)}$ from coherent spanning trees do not cancel. To ensure that $q_{1}^{\delta_{1}} \cdots q_{m}^{\delta_{m}}$ appears in this leading coefficient, we need to show that $\bar{\Sigma}$ has a lift $\Sigma$ in $\Gamma$ that is contained in a coherent spanning tree.

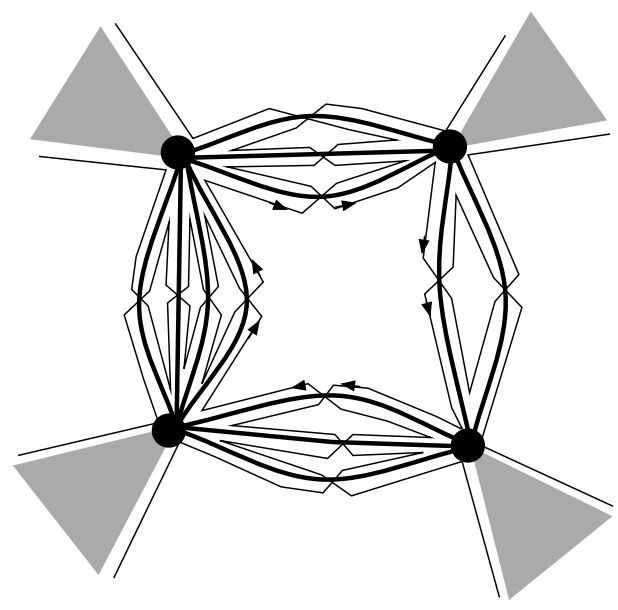

Figure 3: A cycle of anti-parallel twists

Lemma 4.1 Any forest $\bar{\Sigma} \subset \bar{\Gamma}$ of anti-parallel edges extends to a coherent spanning tree $\bar{T}$. 
Proof Let $\bar{\Sigma}$ be a forest consisting of anti-parallel edges. Let $\bar{T}$ be a coherent spanning tree (which exists by our preliminary remarks). By the height of a vertex $v$ in $\bar{T}$ we will mean the distance of that vertex to the root of $\bar{T}$. The root has height 0 .

If some edge $e$ of $\bar{\Sigma}$ is not in $\bar{T}$, then we add it to $\bar{T}$, thereby creating a unique cycle $\bar{C}$. We choose an orientation of $e$ toward the vertex of lower height, if the heights of the two vertices connecting $e$ differ; otherwise, either orientation for $e$ will do. Consequently, $\bar{C}$ consists of two oriented paths. In other words, there is a unique vertex $v_{0}$ in $\bar{C}$ with two outgoing and another vertex $v_{1}$ with two incoming edges. ( $v_{0}$ is actually the vertex $e$ points away from.) Let $x$ be the path in $\bar{C}$ from $v_{0}$ to $v_{1}$ that contains $e$, and let $y$ be the complementary path.

Case 1 The path $y$ consists of edges in $\bar{\Sigma}$. Following the path $x$ from $v_{0}$, let $e^{\prime}$ be the first edge in $x$ contained in $\bar{C} \backslash \bar{\Sigma}$. Such an edge must exist since $\bar{\Sigma}$ is a forest. Delete $e^{\prime}$ and change the orientation of all edges in $x$ that precede $e^{\prime}$, including $e$.

Case 2 The path $y$ has an edge in $\bar{C} \backslash \bar{\Sigma}$. Let $e^{\prime}$ be the first such edge in $y$. Delete $e^{\prime}$ and change the orientation of all edges in $y$ that precede $e^{\prime}$. See Figure 4.

In this way we obtain a new coherent spanning tree, called $\bar{T}$ by abuse of notation, containing one more edge of $\bar{\Sigma}$ than before.

Repeat the procedure until $\bar{\Sigma}$ is contained in $\bar{T}$.

General alternating diagrams $D$ can be expressed as a product $D_{1} * \cdots * D_{r}$ of special alternating (Murasugi) factors. As before, we assume that each of $q_{1}, \ldots, q_{m}$ is greater than 1 .

Recall that each anti-parallel twist appears in some factor. Again consider any monomial $q_{1}^{\delta_{1}} \cdots q_{m}^{\delta_{m}}$ that appears in the leading coefficient of the right-hand side of the equation in Theorem 3.4(2). Its variables can be partitioned so that those in the $i$ th subset arise from an anti-parallel twists in the diagram $D_{i}, 1 \leq i \leq r$. Consider such a subset, say $q_{1}, \ldots, q_{m_{1}}$ after renumbering. The $m_{1}$ twists correspond to anti-parallel edges $e_{1}, \ldots, e_{m_{1}}$ in the graph $\bar{\Gamma}$ corresponding to the special factor $D_{1}$ of $D$. As before, the edges do not form any cycles, and so from them we can form a coherent spanning tree for $\Gamma$. Consequently, $q_{1}^{\delta_{1}} \cdots q_{m}^{\delta_{m_{1}}}$ appears in the leading coefficient of the Alexander polynomial of the Murasugi factor $D_{1}$, and hence it appears in the leading coefficient of $\Delta_{\ell\left(q_{1}, \ldots, q_{n}\right)}$. To finish the proof we remark again that there are finitely many wiring diagrams of order $n$ with no trivial split components, and trivial split components do not alter $M_{e}(\Delta)$. This completes the proof of Theorem 2.1. 


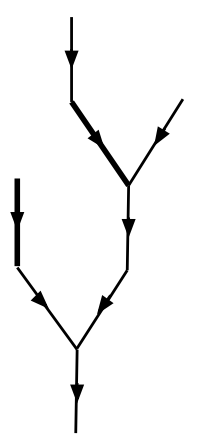

old tree

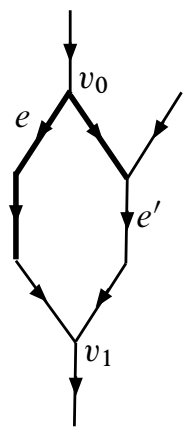

$\longrightarrow$ ordinary edge

$\rightarrow$ anti-parallel edge

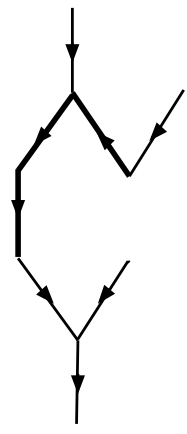

new tree

Figure 4: Exchanging edges $e$ and $e^{\prime}$ in $\bar{T}$

Remark 4.2 Theorem 2.1(2) is true by the same argument for the more general class of homogeneous diagrams defined in [7].

Remark 4.3 One easily observes that the various bounds on polynomial lengths, magnitude and number of non-zero coefficients, and Mahler measure obtained above are (and must be) exponential in the twist number. We have not elaborated on this theme here, but with the inequalities in [31] and a bit extra work, it is possible to give explicit (though likely still not optimal) bounds on the bases of the exponentials.

\section{Examples}

The set of Mahler measures of Alexander polynomials corresponding to knots obtained from a given knot by twisting repeatedly at several sites may have infinitely many limit points. We illustrate this in Example 5.1.

Example 5.1 Consider the family of alternating pretzel knots $\ell(2,2 k+1,2 l+1)$ with $2,2 k+1$ and $2 l+1$ half-twists in first, second and third bands, respectively, $k, l \geq 0$. If we fix $k$ and let $l$ tend toward infinity, then by Theorem 2.2 of [27] the Alexander polynomials of the resulting knots have Mahler measures that approach the limit $M\left(\Delta_{\ell(2,2 k+1, \infty)}(x, z)\right)$, where $\ell(2,2 k+1, \infty)$ is the 2-component link shown in Figure 5 for the case $k=1$.

Algebraic ${ }^{3} \mathcal{G}$ Geometric Topology, Volume 6 (2006) 


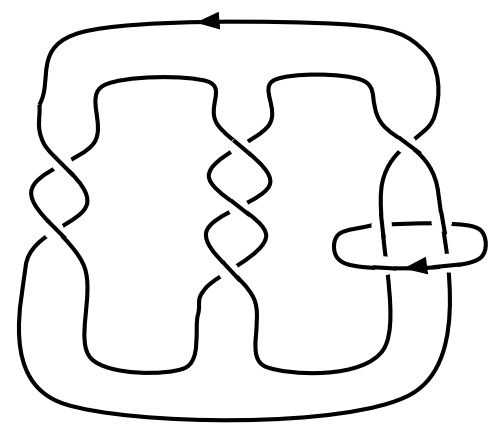

Figure 5: Pretzel link $\ell(2,3, \infty)$

Using Remark 3.3 of [27], we can compute $\Delta_{\ell(2,2 k+1, \infty)}(x, z)$ from the 3-variable Alexander polynomial $\Delta_{\ell(2, \infty, \infty)}(x, y, z)$, where $\ell(2, \infty, \infty)$ is obtained from $\ell(2,2 k+1, \infty)$ by leaving a single half-twist in the second band and encircling it, just as we have done with the third band. We have

$$
\left(x^{2}-1\right) \Delta_{\ell(2,2 k+1, \infty)}(x, z)=\Delta_{\ell(2, \infty, \infty)}\left(x, x^{-2 k}, z\right) .
$$

Since the two polynomials differ only by a cyclotomic factor,

$$
M\left(\Delta_{\ell(2,2 k+1, \infty)}(x, z)\right)=M\left(\Delta_{\ell(2, \infty, \infty)}\left(x, x^{-2 k}, z\right)\right) .
$$

The polynomial $\Delta_{\ell(2, \infty, \infty)}\left(x, x^{-2 k}, z\right)$ can be expressed, up to multiplication by a unit, as

$$
(x-1)\left[\left(1-x-x^{2}-x^{2 k+2}\right)+x^{2 k+4} z\left(1-x^{-1}-x^{-2}-x^{-2 k-2}\right)\right] .
$$

A technique of D Boyd [2] (see [27, Lemma 4.1]) enables us to compute the Mahler measure of this polynomial as the Mahler measure of the single-variable polynomial $f_{k}(x)=1-x-x^{2}-x^{2 k+2}$. An argument based on Rouché's theorem (Brown and Churchill [3]) shows that $f_{k}(x)$ has exactly one zero $\zeta_{k}$ inside the unit circle, a zero that is real. The product of all the moduli of zeros of $f_{k}$ is equal to the modulus of the absolute term 1 , so $M\left(f_{k}\right)=1 /\left|\zeta_{k}\right|$. Since for $k \neq k^{\prime}$ the difference $f_{k}(x)-f_{k^{\prime}}(x)$ is a unit times a cyclotomic polynomial, $f_{k}$ and $f_{k^{\prime}}$ have no common zeros off the unit circle. In particular, $\zeta_{k} \neq \zeta_{k^{\prime}}$ whenever $k \neq k^{\prime}$. Now at most two real numbers have the same modulus, thus the Mahler measures of the polynomials $f_{k}$ are triplewise distinct, and so the set of values $M\left(\Delta_{\ell(2,2 k+1,2 l+1)}(x)\right)$ has infinitely many distinct limit points.

Theorem 2.1 implies that the limit points are bounded. 
Example 5.2 shows that the conclusion of Theorem 2.1(2) does not hold if the hypothesis that the diagrams be alternating is dropped.

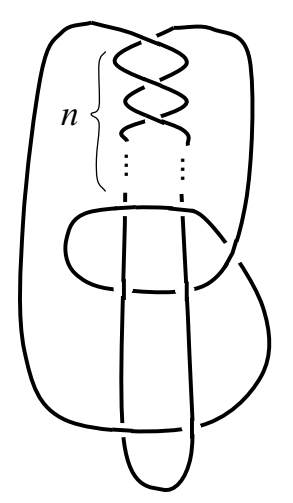

Figure 6: Non-alternating knot diagram $D_{n}$

Example 5.2 Consider the family of non-alternating diagrams $D_{n}$ in Figure 6, each member containing $n=1,3,5, \ldots$ half-twists in its upper portion. The Alexander polynomials of the corresponding knots $k_{n}$ are easily seen to be

$$
\Delta_{k_{n}}(t)=t^{4}-\frac{n+5}{2} t^{3}+(n+4) t^{2}-\frac{n+5}{2} t+1 .
$$

The twist numbers of the diagrams $D_{n}$ are all equal. However, the euclidean Mahler measures of the polynomials must tend to infinity, since the sum of the four roots is $(n+5) / 2$ (and so one root has modulus at least $(n+5) / 8)$. Since the volumes of $k_{n}$ are also bounded (by Thurston's hyperbolic surgery theorem; see Lackenby [15]), similarly Corollary 2.3 also fails without the alternation assumption.

We conclude by showing that the converse of Theorem 2.1(2) does not hold.

Example 5.3 Consider the tangle $T$ in Figure 7. By replacing a single crossing by $T$ in the diagram of a trefoil, as in the figure, we obtain the knot $8_{10}$. Iterating the procedure, always replacing (for the sake of definiteness) the top crossing, we obtain a sequence of diagrams $D_{n}$ for alternating knots $k_{1}=3_{1}, k_{2}=8_{10}$, et cetera.

We claim that $\Delta_{k_{n}}(t)=\left(t-1+t^{-1}\right)^{2 n-1}$, for each $n$. Consider the Conway skein module over the field of fractions of $\mathbb{Z}\left[t^{1 / 2}, t^{-1 / 2}\right]$ generated by oriented tangles modulo the relations $\ell_{+}-\ell_{-}-\left(t^{1 / 2}-t^{-1 / 2}\right) \ell_{0}$, where $\left(\ell_{+}, \ell_{-}, \ell_{0}\right)$ is any skein 

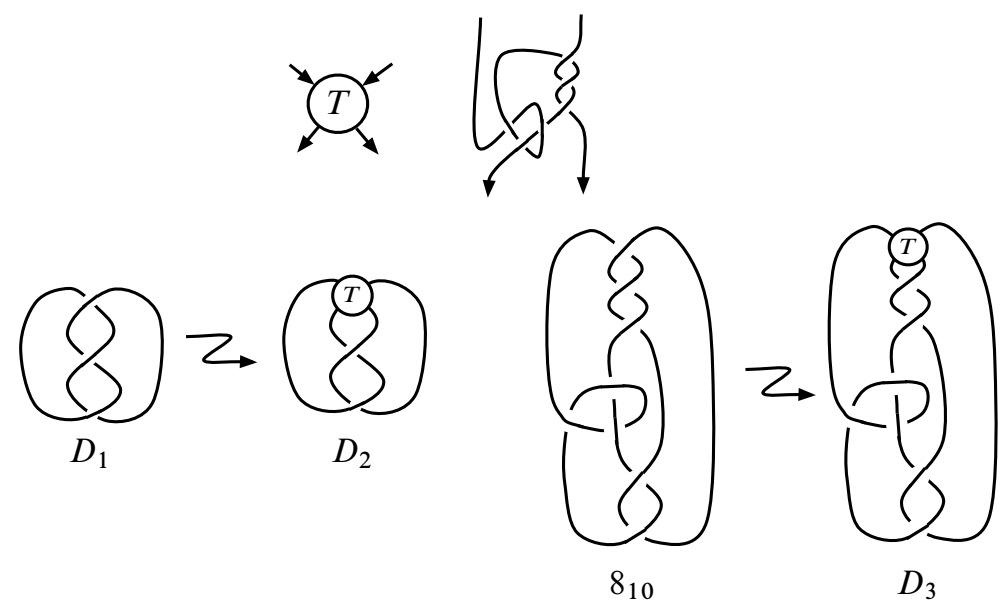

Figure 7: Tangle $T$ and alternating knot diagrams

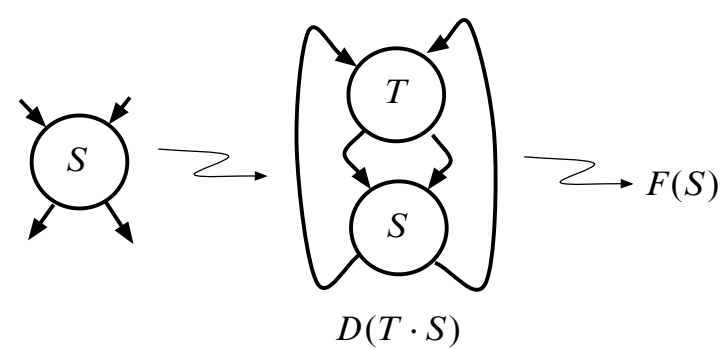

Figure 8: Linear functional $F$

triple. The tangle $T$ induces a mapping $F: S \mapsto F(S)=\Delta_{D(T \cdot S)}(t)$, where $D(T \cdot S)$ denotes the denominator closure of the tangle product of $T$ and $S$ (see Figure 8).

The tangles $S_{1}, S_{2}$ in Figure 9 form a basis for the skein module. We can write $T=f(t) S_{1}+g(t) S_{2}$, for some scalars $f(t), g(t) \in \mathbb{Z}\left[t^{1 / 2}, t^{-1 / 2}\right]$. Then

$$
F(S)=f(t) \cdot \Delta_{D\left(S_{1} \cdot S\right)}(t)+g(t) \cdot \Delta_{D\left(S_{2} \cdot S\right)}(t),
$$

for any tangle $S$.

We can find $f(t)$ and $g(t)$ easily by substituting proper values for $S$ in (5-1). If $S=S_{1}$, then $D(T \cdot S)$ is the square knot, $D\left(S_{1} \cdot S\right)$ is a trivial 2-component link, and $D\left(S_{2} \cdot S\right)$ is an unknot. Hence $\left(t-1+t^{-1}\right)^{2}=f(t) \cdot 0+g(t) \cdot 1$, and so 


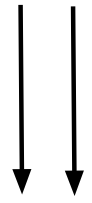

$S_{1}$

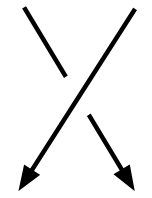

$S_{2}$

Figure 9: Basis $S_{1}, S_{2}$

$g(t)=\left(t-1+t^{-1}\right)^{2}$. Similarly, if $S=S_{2} \cdot S_{2}$, then $D(T \cdot S)$ is the knot $8_{10}$, $D\left(S_{1} \cdot S\right)$ is a Hopf link, and $D\left(S_{2} \cdot S\right)$ is a trefoil. So

$$
\begin{aligned}
\Delta_{8_{10}} & =\left(t-1+t^{-1}\right)^{3}=\left(t^{1 / 2}-t^{-1 / 2}\right) \cdot f(t)+\left(t-1+t^{-1}\right) \cdot g(t) \\
& =\left(t^{1 / 2}-t^{-1 / 2}\right) \cdot f(t)+\left(t-1+t^{-1}\right)^{3} .
\end{aligned}
$$

Thus $f(t)=0$, and in the skein module $T=\left(t-1+t^{-1}\right)^{2} S_{2}$, and then $\Delta_{k_{n}}=$ $\left(t-1+t^{-1}\right)^{2} \Delta_{k_{n-1}}$. Since $\Delta_{k_{1}}(t)=t-1+t^{-1}$, we have $\Delta_{k_{n}}(t)=\left(t-1+t^{-1}\right)^{2 n-1}$.

Observe that the diagrams in Figure 7 have unbounded twist number (and hence, by [15] also volume), while the Alexander polynomials $\Delta_{k_{n}}(t)$ are all products of cyclotomic polynomials and hence have trivial Mahler measure.

Remark 5.4 If one drops the alternation assumption, then for any knot $k$ there exists a hyperbolic knot $\tilde{k}$ with arbitrarily large volume (and twist number) and the same Alexander polynomial as $k$. For trivial polynomial knots this result was proved in [13]. Another method that constructs for any (admissible) Alexander knot polynomial (in fact, Alexander invariant) an arborescent knot $\tilde{k}$ is given by the Stoimenow in [28]. Silver and Whitten [24] have used a yet different construction to choose $\tilde{k}$ so that the group of $\tilde{k}$ can be mapped onto the group of $k$ sending meridian to meridian and longitude to longitude.

\section{References}

[1] S Bhatty, Mahler measure and the Alexander polynomial of pretzel links, Undergraduate honors thesis (S G Williams, advisor) University of South Alabama, Mobile AL (2004)

[2] D W Boyd, Speculations concerning the range of Mahler's measure, Canad. Math. Bull. 24 (1981) 453-469 MR644535 
[3] J W Brown, R V Churchill, Complex variables and applications, McGraw-Hill Book Co., New York (1984) MR730937

[4] P J Callahan, J C Dean, J R Weeks, The simplest hyperbolic knots, J. Knot Theory Ramifications 8 (1999) 279-297 MR1691433

[5] A Champanerkar, I Kofman, On the Mahler measure of Jones polynomials under twisting, Algebr. Geom. Topol. 5 (2005) 1-22 MR2135542

[6] A Champanerkar, I Kofman, E Patterson, The next simplest hyperbolic knots, J. Knot Theory Ramifications 13 (2004) 965-987 MR2101238

[7] P R Cromwell, Homogeneous links, J. London Math. Soc. (2) 39 (1989) 535-552 MR1002465

[8] O Dasbach, X-S Lin, A volume-ish theorem for the Jones polynomial of alternating knots arXiv:math.GT/0403448

[9] G Everest, T Ward, Heights of polynomials and entropy in algebraic dynamics, Universitext, Springer London Ltd., London (1999) MR1700272

[10] C M Gordon, Toroidal Dehn surgeries on knots in lens spaces, Math. Proc. Cambridge Philos. Soc. 125 (1999) 433-440 MR1656809

[11] E Hironaka, The Lehmer polynomial and pretzel links, Canad. Math. Bull. 44 (2001) 440-451 MR1863636

[12] V F R Jones, Hecke algebra representations of braid groups and link polynomials, Ann. of Math. (2) 126 (1987) 335-388 MR908150

[13] E Kalfagianni, Alexander polynomial, finite type invariants and volume of hyperbolic knots, Algebr. Geom. Topol. 4 (2004) 1111-1123 MR2113898

[14] K Kodama, Knot, a program for computing knot invariants, available at http://www.math.kobe-u.ac.jp/ kodama/knot.html

[15] M Lackenby, The volume of hyperbolic alternating link complements, Proc. London Math. Soc. (3) 88 (2004) 204-224 MR2018964 (with an appendix by Ian Agol and Dylan Thurston)

[16] D H Lehmer, Factorization of certain cyclotomic functions, Ann. of Math. (2) 34 (1933) 461-479 MR1503118

[17] D A Lind, T Ward, Automorphisms of solenoids and p-adic entropy, Ergodic Theory Dynam. Systems 8 (1988) 411-419 MR961739

[18] K Mahler, On some inequalities for polynomials in several variables, J. London Math. Soc. 37 (1962) 341-344 MR0138593

[19] K Murasugi, On alternating knots, Osaka Math. J. 12 (1960) 277-303 MR0137107

[20] K Murasugi, Non-amphicheirality of the special alternating links, Proc. Amer. Math. Soc. 13 (1962) 771-776 MR0142116 
[21] K Murasugi, J H Przytycki, The skein polynomial of a planar star product of two links, Math. Proc. Cambridge Philos. Soc. 106 (1989) 273-276 MR1002540

[22] K Murasugi, A Stoimenow, The Alexander polynomial of planar even valence graphs, Adv. in Appl. Math. 31 (2003) 440-462 MR2001624

[23] H Seifert, Über das Geschlecht von Knoten, Math. Ann. 110 (1935) 571-592 MR1512955

[24] D S Silver, W Whitten, Hyperbolic covering knots, Algebr. Geom. Topol. 5 (2005) 1451-1469 MR2186104

[25] D S Silver, S G Williams, Lehmer's question, knots and surface dynamics arXiv: math.GT/0509068

[26] D S Silver, S G Williams, Mahler measure, links and homology growth, Topology 41 (2002) 979-991 MR1923995

[27] D S Silver, S G Williams, Mahler measure of Alexander polynomials, J. London Math. Soc. (2) 69 (2004) 767-782 MR2050045

[28] A Stoimenow, Alexander polynomials and hyperbolic volume of arborescent links, preprint

[29] A Stoimenow, A property of the skein polynomial with an application to contact geometry arXiv:math.GT/0008126

[30] A Stoimenow, The Jones polynomial, genus and weak genus of a knot, Ann. Fac. Sci. Toulouse Math. (6) 8 (1999) 677-693 MR1815161

[31] A Stoimenow, On the coefficients of the link polynomials, Manuscripta Math. 110 (2003) 203-236 MR1962535

DSS, SGW: Department of Mathematics and Statistics, University of South Alabama Mobile, AL 36688-0002, USA

AS: Graduate School of Mathematical Sciences, University of Tokyo

3-8-1, Komaba, Tokyo 153-8914, Japan

silver@jaguar1.usouthal.edu, stoimeno@ms.u-tokyo.ac.jp,

swilliam@jaguar1. usouthal. edu

Received: 26 March 2005 